\title{
Ueber die Bildung von Krystallen mit Kermen;
}

\section{von Hermann Kopp.}

Bei Krystallen zeigt sich bekanntlich nicht selten, dafs sich darin ein Kern von der umgebenden Hülle nach Farbe oder Durchsichtigkeit unterscheiden läfst. Der Kern kann dieselbe Form oder eine andere zeigen, wie die ihn ungebende Hülle. Unter den Mineralien sind namentlich für den Flufsspath viele hierher gehörige Fälle bekannt geworden. Da bei solchen Krystallen die Spaltungsrichtungen gleichmäfsig sich fortsetzend durch die Hülle und den Kern gehen, ist die Ausbildung der Krystalle eine stetige gewesen. Die Krystallbildung hatte z. B. zuerst unter Umständen statl, wo Beimischungen in der Flüssigkeit, aus welcher die Krystallbidung vor sich ging, besondere Form und Färbung bedingten; nach Entfernung jener Beimischungen aus der Flüssigkeit oder dem Zutreten einer von solchen Beimischungen freien Flüssigkeit zu den erst gebildeten Krystallen lagerte sich die Hülle unmiltelbar an den zuvor auskrystallisirten Kern an. Solche Krystallbildungen lassen sich leicht nachahmen; ein Octaëder von mittelst wenig Chromalaun amethyslfarben gefärbtem gewöhnlichem Alaun überzieht sich z. B. bekanntlich in gesättigter Alaunlösung mit einer farblosen Hülle.

Weniger deutlich könnte auf den ersten Blick die Bildung solcher Krystalle erscheinen, wo Kern und Hülle so leicht von einander trennbar sind, dafs man glauben, könnte, sie seien gar nicht zusammen verwachsen. Das ist bei den $\mathbf{s . g}$. schalig zusammengesetzten Krystallen der Fall, wo sich oft die Hülle von dem Kern abheben läfst, und sich auch nicht selten noch ein feiner Staub zwischen Hülle und Kern findet.

Meine Aufmerksankeit wurde diesem Gegenstand vor längerer Zeit durch die Untersuchung von Kalkspathkrystallen 
zugewendet, welche auf einem Stück Kalkstein von Kleinlinden bei Giefsen aufgewachsen waren. Die mehrere Millimeter grofsen Krystalle waren schlecht ausgebildet; sie zeigten vorherschend 4R, an den Enden durch ein Scalenoëder mit rauhen Flächen zugespitzt. Bei der Ablösung von dem Kalkstein trennte sich leicht die äufsere Masse des Krystalls von einem eingeschlossenen Kern, einem scharf ausgebildeten Scalenoëder $+R^{3}$; dieser Kern war mit röthlichem Sediment überzogen. Ich habe später noch andere Kalkspathformen beobachtet, in welchen auch $+R^{3}$ als leicht herauszunehmender Kern enthalten war.

Ein Flufspath aus Derbyshire in meiner Sammlung zeigt einen Würfel mit etwa $14^{\mathrm{mm}}$ langen Kanten. Im refleclirten Lichte betrachtel scheint er einen Schwefelkieswürfel mil etwa $12^{\mathrm{mm}}$ langen Kanten als parallel gestellen Kern eingeschlossen zu enthalten; im durchgelassenen Licht sieht man, dafs die metallglänzende Substanz, welche im zurückgeworfenen Lichte wie ein Schwefelkieskrystall aussieht, nur sehr dünne Schichten bildet. Hier wurde ein Würfel von Flufsspath mit einer fremdartigen Substanz, die sich darauf abselzte, überzogen, und dann fand eine neue Krystallisation von Flufsspath statt, welche die Hülle zu jenem Kern abgab.

Solche Fälle könnten vermuthen lassen, die Krystallisationskraft könne auf einige Entfernung wirken. Das wäre allerdings dann der Fall, wenn ein schon gebildeter Kern die Lage sich aus einer Flüssigkeit ausscheidender Moleküle bedingte und diese symmetrisch gestellt um ihn sich anlagern liefse, ohne dafs sie wirklich mit ihm zusammenwachsen.

Wenn auch Fälle, wie die oben angeführten, hierfür zu sprechen scheinen, ist eine solche Wirkung der Krystallisationskraft doch höchst unwahrscheinlich, und bei zahlreichen Versuchen, wo ich dem oben erwähnten natürlichen Vorkommen von schalig zusammengesetzten Krystallen ganz 
entsprechende künstliche Producte darstellte, zeigte sich auch nie eine Erscheinung, welche, aufmerksam verfolgt, für eine solche Wirkung spräche.

Taucht man einen Alaunkrystall in mit ätherischem Alkannaauszug gefärbte Collodionlösung, so überzieht er sich dann an der Luft schnell mit einer rothen Schichte. Legt man einen solchen, mit Collodion überzogenen Krystall in eine frisch gesättigte Alaunlösung, und überläfst diese der langsamen Verdunstung, so ist nach einiger Zeit der mit rothgefärbtem Collodion überzogene Alaunkryslall mit einer durchsichtigen Hülle von Alaun umkleidet. Man hat dann einen wohlausgebildeten Alaunkrystall mit rothem Kern, welcher, wenn man ein Eck des Octaëders mittelst einer feinen Säge abnimmt, die schalige Zusammensetzung vollkommen zeigt; die äufsere Hülle läfst sich von dem rothen Kern ganz leicht abnehmen.

Doch findet hier keine Wirkung der Krystallisationskraft durch die Collodionschichte hindurch statt. Beobachtet man einen mit Collodion überzogenen Alaunkrystall, der in eine Alaunlösung gelegt wurde, welche für eine um einige Grade höhere Temperatur als die der Luft gesättigt wurde, so sieht man an einzelnen Stellen, wo die Collodionschichte offenbar den Krystallkern nicht vollständig deckt, namentlich an den Kanten, einzelne kleine Kryställchen sich anlegen. Diese wachsen und bilden Stäbe längs den Kanten des Kernkrystalls; von diesen Stäben aus überwächst der ganze Krystall und es bildet sich allmälig eine vollständige durchsichtige Hülle.

Eine Hülle kann somit um einen Kern sich ausbilden, welcher mit einer fremdartigen Substanz überzogen ist, wenn letatere auch nur an wenigen Punkten das unmittelbare Anwachsen einiger Krystallmoleküle an den eingeschlossenen Krystall gestattet. Die Hülle kann sich defswegen vollständig 
und ganz regelmäfsig von diesen, zuerst zerstreut und in geringer Anzahl anwachsenden Kryställchen aus ausbilden, weil diese sämmllich durch den eingewachsenen Krystall in parallele Stellung orientirt sind *). Die Zahl der Beruhrungspunkte zwischen Kern und Hülle, welche zur Ausbildung der letzteren nothwendig sind, ist so klein, dafs dadurch bei der Trennung der Hülle von dem Kern kein bemerkbares Hindernifs hervorgebracht wird.

Man kann ebenso auch einen Würfel von Alaun mit rothgefärbtem Collodion überziehen und in einer gewöhnlichen Alaunlösung mit einer Hülle überwachsen lassen. Man erhält ein farbloses Octaëder, welches einen rothen Würfel als Kern schalig eingeschlossen enthält. Der Kern scheint auch hier bei der Zerkleinerung des Krystalls mit der Hülle nicht zusammengewachsen zu sein; er ist es indefs doch, freilich nur an sehr wenigen Punkten.

Ich will hier noch Einiges über die Bildung von Krystallen mit durchaus damit zugewachsenen Einschlüssen anführen.

*) Ohne Zweifel beruhi auf ähnlichen Umständen die Bildung von Krystalten, welche eine grofse Menge fremder Substanz mechanisch beigemengt enthalten. Hierher gehörige Fălle sind nicht selten beobachtet worden; das bekannteste Beispiel ist das Vorkonımen von Kalkspathkrystallen, welche grofse Mengen Quarzsand enthalten. Auch hier ist die Ausbildung grofser Krystalle dadurch möglich, dafs von dem ersten Entstehen des Krystalls an sich alle Kalkspaththeilchen, die Sandkörner umschliefsend, in paralleler Stellung an einander lagern. - Dafs solche Krystalle Gemenge von zwei Substanzen sind, ist dann leicht zu erkennen, wenn die eine Substanz z. B. in Säuren leicht löslich ist, die andere nicht. Aber eskönnen in derselben Weise Substanzen zusammenkrystallisiren, welche beide nur durch Flufssäure oder durch kohlensaures Natron aufschliefsbar sind und wo die Analyse nur die Durchschnittszusammensetzung des mechanischen Gemenges ergeben kann. Es gereicht dem, was in der Lehre vom Isomorphismus sicher begründet ist, nicht xum Nachtheil, in solchen Fállen keine Erklärung für die Beziehungen zwischen Zusammensetzung und Form geben und keine wabrscheinlichen Formeln anfstellen zu können. 
Es ist noch in der neueren Zeit behauplet, aber auch widerlegt worden, der cubische Alaun habe eine andere $\mathrm{Zu}$ sammensetzung als der octaëdrische. Dabei stützte man sich auch auf die physikalischen Verschiedenheiten, die Undurchsichtigkeit des cubischen und die Durchsichtigkeit des octaëdrischen Alauns. - Aus mit etwas Kali versetzter Alaunlösung krystallisiren Würfel, die zuerst durchsichtig sind, beim Wachsen undurchsichtig werden. Letzteres rührt nicht allein davon her, dafs etwas von dem Niederschlag, welcher sich in mit etwas Kali versetztęr Alaunlösung beim Stehen an der Luft abscheidet, bei dem Wachsen der Krystalle mechanisch eingeschlossen wird, sondern in einer auf die Würfelflächen rechtwinkeligen Richtung betrachtet ist der Alaun überhaupt oft weniger durchsichtig, als in einer auf die Oclaëderflächen rechtwinkeligen Richtung betrachtet. An Mittelkrystallen von Octaëder und Würfel sah ich öfters in der ersteren Richtung den Krystall vollkommen undurchsichtig, in der zweiten Richtung durchsichtig oder doch sehr stark durchscheinend. Wo man so den undurchsichligen cubischen Alaun und den durchsichtigen octaëdrischen in Einem Krystall hat, beantwortet sich die Frage über eine etwaige Verschiedenheit der Zusammensetzung beider von selbst. Es ist mir auch nicht gelungen, einen Krystall von Alaun hervorzubringen, der als Hülle ein durchsichtiges Octaëder, als Kern einen undurchsichtigen Würfel zeige. Bei dem Wachsen eines undurchsichtigen Würfels von Alaun in einer gewöhnlichen Alaunlösung geht das Wachsen in der Art vor sich, dafs die Krystallflächen stels eben bleiben, aber mit stetiger Verkleinerung der Würfelflächen; zuerst entsteht ein Würfel mit abgestumpften Ecken, dann der Mittelkrystall, dann das vorherrschende Octaëder, in welchem letztern aber kein würfelförmiger Kern deutlich zu sehen ist. 
Anders wächst der Chromalaun auf einen Würfel von gewöbnlichem Alaun an. Der Chromalaun scheint nicht mit vorherrschenden Würfelflächen krystallisiren zu können. In einer bei sehr wenig erhöhter Temperatur gesättigten und langsam abkühlenden Chromalaunlösung überzieht sich ein Würfel von Thonerdealaun mit einer zahllosen Menge Octaëder von Chromalaun, welche seine Flächen rauh und mit kleinen Spitzen übersäet erscheinen lassen. Alle diese Octaëder sind aber durch den Würfel zu paralleler Stellung orientirt und wachsen allmälig, bei dem Verdunsten der Chromalaunlösung, zu Einem regelmälsigen Octaëder mit glatten Flächen zusammen, welches den Würfel von Thonerdealaun als Kern enthält; ein Durchschnitt parallel einer Würfelfläche zeigt ein weifses Quadrat (den Thonerdealaun) diagonal in ein dunkel violettes (den Chromalaun) eingeschlossen.

Es ist merkwürdig, dafs Chromalaun und Thonerdealaun, welche als isomorphe Körper sich hinsichtlich der kírystallisationswirkungen ziemlich einerlei verhalten und einander ergänzend und vergrö fsernd krystallisiren, doch nur mit sehr geringer Cohäsion zusammenwachsen. Läfst man über ein Oclaëder von Chromalaun, dessen Flächen mit Nichts Fremdartigem in Berührung kamen, eine Hülle von gewöhnlichem Alaun wachsen, so läfst sich diese leicht auf grofse Strecken hin in Einem Stück von dem Chromalaun ablösen. Man thut wohl, dieses zu beachten, wenn man, zur Demonstration des Uebereinanderwachsens isomorpher Körper, Chromalaun und Thonerdealaun in abwechselnden Schichten über einander wachsen lärst und dann, un die Reihenfolge der Schichten zu zeigen, miltelst einer feinen Säge ein Eck des Octaëders parallel einer Würfelfläche abschneidet und die Schniltfläche eben schleift; man mufs die äufserste Schichte ziemlich dick wachsen lassen, damit sie bei diesen 
Operationen nicht von der darunter liegenden abbreche, und das darzustellende Krystallpräparal die Schnittfläche gut begrenzt zeige *).

Es ist, auch noch in neuerer Zeit, behauptet worden, die Krystalle könnten durch ihr Bestreben zu wachsen mechanische Effecte ausüben und z. B. ein Hindernifs mechanisch beseitigen, welches ihrer Ausbildung entgegensteht. So unwahrscheinlich dieses ist, stellte ich doch einige Versuche auch in dieser Richtung an. Ein Krystall von nur wenig gröfserem spec. Gewicht, als das der Lösung, in welcher er wächst, drückt nur mit sehr geringer Kraft auf seine Unterlage, und es wäre nach jener Ansicht wohl zu erwarten, dafs der Krystall beim Wachsen diesen geringen Druck überwinde und sich etwas hebe, um auch nach unten zu wachsen. Lälst man einen durch eine geringe Menge Chromalaun gefärbten Alaunkrystall in einer reinen Alaunlösung wachsen, so kann man sehr deutlich sehen, wo an

*) Um sehr grofse Krystalle zu ziehen, ist es vortheilhaft, einen ganz kleinen, möglichst regelmäfsig ausgebildeten Krystall symmetrisch mittelst etwas Wachs au eine Glasröhre von einigen Millimeter Durchmesser zu befestigen (so dafs z. B. bei einem Alaunkrystall eine octaëdrische Axe in die Richtung der Axe der Glasröhre kommt) und ihn so in die unterste Schichte einer gesăttigten Lósung zu hängen, die man nur, wenn die Temperatur rasch steigt, von Neuem săıtigt. Bei dem langsamen Verdunsten der Losung whichst der Krystall sehr regelmâfsig und legt sich um die Glasróhre herum fest an, welche später zum Aufstellen des Krystalls dient. Auf diese Weise erhält man leicht sehr regelmâfsig ausgebildete grofse Krystalle, während diese auf einer Flăche aufliegend selbst bei ơfterem Wechseln dẹrselben gewöhnlich verzerrt ausfallen. Die Gewinnung von grofsen Octaëdern (bis zu 60 oder $70 \mathrm{~mm}$ Kantenlänge) von Thonerdealaun oder Chromalaun oder öfters abwechselnden Schichten beider Alaune hat auf diese Art keine Schwierigkeit. 
ihm Vergrölserung stattfindet; ich konnte indessen nie etwas beobachten, was die Ansicht unterstützte, dafs ein Krystall sich heben kann, um auch an der Fläche, mit welcher or aufsitzt, zu wachsen.

\section{Ueber die Darstellung des Ferrum pulveratum.}

Die ärztliche Erfahrung hat gezeigt, dafs das metallische Eisen in fein zertheiltem Zustand eine der wirksamsten Formen ist, in welchen dieses Metall als Arzneimittel angewendet wird. Bisher wurde dazu mechanisch fein pulverisirtes Stabeisen unter dem Namen Ferrum pulveratum genommen; aber schon 1840 schlugen Quevenne und Miquelard dazu das durch Wasserstoffgas aus dem 0xyd reducirte Eisen vor ${ }^{*}$, und in dieser Form und diesen Zustand von vollkommener Reinheit scheint es in der That wesentliche Vorzüge vor dem gewöhnlichen, minder leicht lüslichen, kohlehaltigen Stabeisenpulver zu haben. Auch wird es jetzt in Frankreich unter dem Namen Fer réduit allgemein angewendet. Die Darstellung ist eine ganz leichte, in jeder Apotheke ausführbare Operation; jedenfalls hat sie für den Fabrikanten pharmaceutischer Präparate nicht die geringste Schwierigkeit. Das Umständlichste dabei ist die Bereitung des dazu erforderlichen reinen Eisenoxyds, wenn man dieses durch Fällung eines Eisenoxydsalzes mit Ammoniak darstellen will. Allein diefs hat man nicht nöthig, sondern man verfährt auf folgende Weise :

*) Memoire sur l'action physiologique et thérapeutique des ferrugineux; par $\mathbf{Q}$ uevenne, in den Archives de physiologie sous la direction de Bouchardat. Nr. 2, October 1854. 